\title{
Influence du coefficient d'uniformité sur l'amplitude et le taux de l'affaissement des sols
}

\section{T. AYADAT}

PhD en Génie Civil, enseignant à I'Université

de M'Sila

B. BELOUAHRI

Magister en Génie Civil, enseignant à l'Université de M'Sila
La plupart des régions arides et semi-arides du monde possèdent des dépôts superficiels de sols granulaires lâches. Certains de ces sols sont susceptibles de subir une grande réduction dans leur volume, après mouillage de leur structure, en donnant des tassements exorbitants. De tels dépôts sont connus comme des sols affaissables (collapsibles soils).

La majorité des travaux effectués sur les paramètres qui gouvernent l'affaissement de ces sols ont été consacrés à la densité sèche initiale, la teneur en eau, le degré de saturation et la charge appliquée. Comparativement, très peu de travaux ont été orientés vers l'étude de l'influence de la granulométrie sur le comportement de ces sols. Cette recherche expérimentale consiste à illustrer l'influence du coefficient d'uniformité (ou coefficient de Hazen) sur le taux et l'amplitude de l'affaissement.

\section{Influence of uniformity coefficient on the magnitude and rate of collapse}

Many arid and semi-arid areas of the world have deposit of loose granular soils. Some of these soils are prone to undergo large reduction in volume when they wet up giving rise to significant settlements. Such deposits are known as \& collapsing soils $x$.

Most of the works that have been performed on the parameters governing collapse have concentrated on the initial dry density, moisture content, degree of saturation and overburden pressure. Comparatively little work has been carried out in order to investigate the influence of soil grading on the collapse behaviour. This paper illustrate the influence of the uniformity coefficient on the rate and the amount of collapse. 


\section{NOTATIONS}

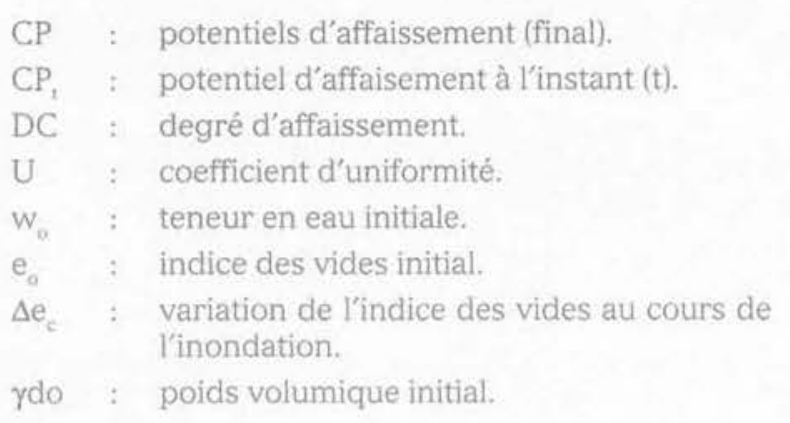

\section{1}

\section{Introduction}

Des désordres importants causés par l'effondrement des sols ont été constatés dans de nombreuses régions du monde, notamment quand elles sont arides ou semi-arides. Ce problème spécial, qui est une réalité sur le terrain, nécessite de la part du géotechnicien une attention particulière et la maîtrise du phénomène.

Les sols affaissables ou «collapsibles» sont définis comme des structures partiellement saturées, pouvant subir un réarrangement radical de leurs particules, suivi d'une grande diminution brusque de leur volume, après avoir été inondées à l'eau avec ou sans surcharge.

Ces sols n'ont fait l'objet d'étude que ces dernières années, bien que Terzaghi fut le premier a avoir reconnu leur existence. En effet, dans les régions arides où on les a souvent localisé, il y a eu un véritable développement de l'industrie, de l'agriculture et de l'urbanisme grâce aux progrès récents de l'irrigation. Ceux-ci impliquent évidemment, l'utilisation de grandes quantités d'eau.

Les tassements excessifs qui en résultent ont donné une impulsion à l'étude de ces sols. Certains chercheurs se sont penchés sur les méthodes d'identification et de traitement, Jennings et Knight (1975), etc., d'autres études ont été consacrées aux mécanismes de l'affaissement: Knight (1961); Barden et al. (1969), etc.

Généralement, une multitude de facteurs comme; le type de sol (minéralogie et granulométrie), la charge appliquée, le degré de saturation, la nature des agents de cimentation, la nature chimique du liquide se trouvant dans les pores et le débit d'eau qui inonde ces sols (dû à la remontée d'une nappe où l'infiltration des eaux de pluie, etc.), contrôlent le potentiel d'affaissement de ces sols.

La plupart des travaux de recherche qui ont été menés sur les paramètres qui gouvernent l'affaissement, se sont concentrés sur la densité sèche initiale, la teneur en eau, le degré de saturation et la charge appliquée. L'influence de ces paramètres sur l'affaissement a été étudiée par plusieurs chercheurs et bon nombre d'entre eux s'accordent sur les points suivants:

1) pour une teneur en eau donnée, le potentiel d'affaissement décroît avec l'augmentation de la densité sèche initiale et avec l'augmentation de la teneur en eau; le potentiel d'affaissement décroît jusqu'à s'annuler lorsque la teneur en eau dépasse une certaine valeur critique (Barden et al., 1969; Lawton, 1989);

2) pour une densité sèche donnée, la surcharge pour laquelle le potentiel d'affaissement est maximal

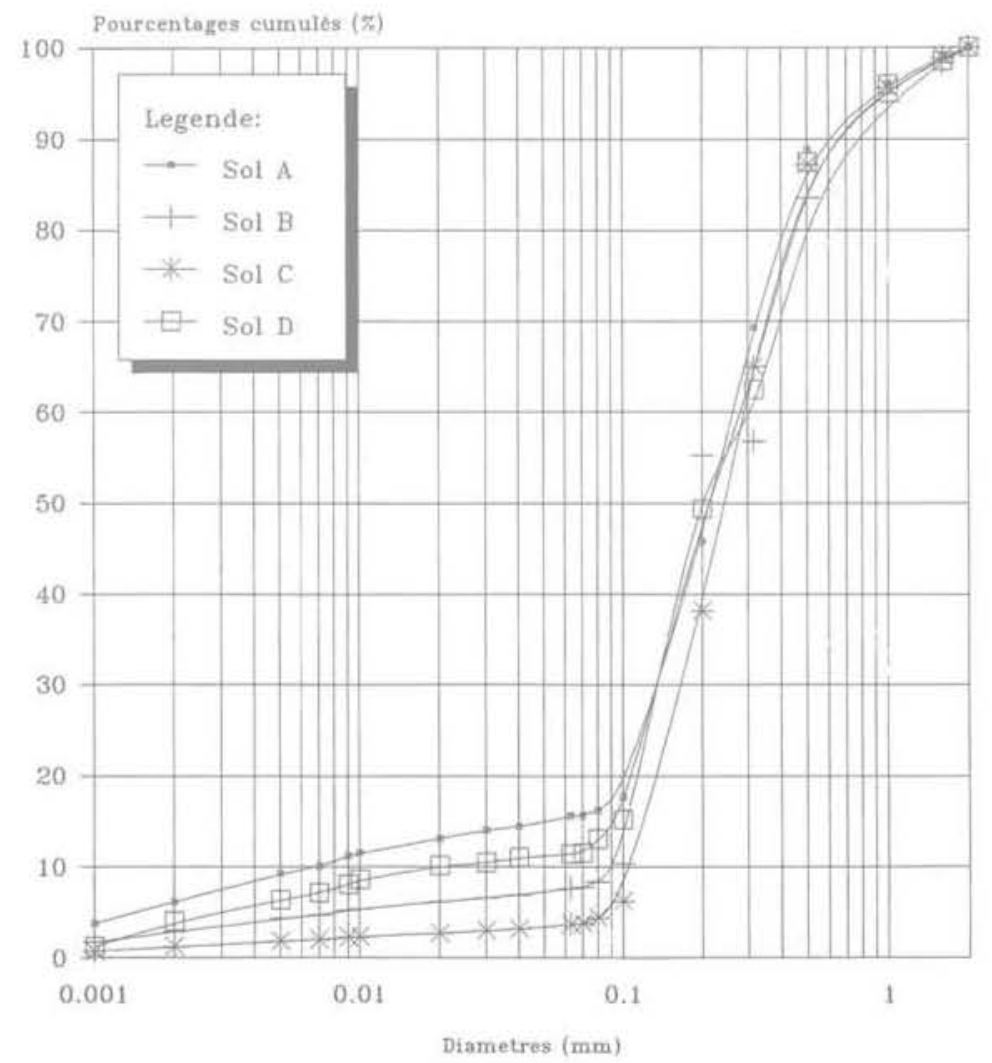


varie inversement avec la teneur en eau du sol (Lawton, 1989):

3) il existe un degré de saturation critique, au-delà duquel les sols ne sont plus susceptibles d'affaissement. Booth (1977) a proposé un degré de saturation critique entre 50 et $60 \%$. Markin (1969) et Prusza, Choudry (1979) ont suggéré des valeurs légèrement supérieures du degré de saturation, entre 60 et $65 \%$.

En ce qui concerne l'influence de la granulométrie du sol sur l'amplitude et le taux d'effondrement, très peu de travaux lui ont été consacrés. Bull (1964) a montré que le tassement maximal peut se produire, lorsque la proportion d'argile est de $12 \%$ des grains solides du sol. Au-dessous de $5 \%$, on observe un faible tassement et au-dessus de $30 \%$, l'argile gonfle. Entre ces limites, il $y$ a des cas où les sols ayant une grande porosité tassent plus que les sols qui ont le même indice des vides initial $\left(\mathrm{e}_{\mathrm{c}}\right)$, mais avec des pores plus petits.

En revanche, Handy (1973) suggère que pour une teneur en argile inférieure à $16 \%$, il y a une grande probabilité d'affaissement; entre $16 \%$ et $24 \%$, l'affaissement est probable; entre $24 \%$ et $32 \%$, la probabilité d'effondrement du sol est inférieure à $50 \%$. Au-dessus de $32 \%$ d'argile, le sol est en général non susceptible d'affaissement.

On note que, malgré le voisinage de ces deux critères, aucun n'est tout à fait assuré de prévoir l'affaissement d'un sol particulier. La question qui se pose est la suivante: est-ce qu'un sol de granulométrie uniforme, possédant une teneur en argile comprise entre $5 \%$ et $30 \%$ a tendance à se comporter comme un sol affaissable?

Anderson (1968) a proposé une équation pour identifier les sols affaissables de l'Arizona (USA). Cette approche empirique fait intervenir, entre autres, le coefficient d'uniformité, ainsi que les diamètres des particules correspondants aux différents pourcentages en poids du sol. Celle-ci ne donne pas une image claire de l'influence de la granulométrie sur l'affaissement.

Cet article a pour but d'illustrer l'influence de la granulométrie (coefficient d'uniformité) sur le taux et l'amplitude de l'affaissement.

\section{2}

\section{Matériaux - Matériels - Essais}

\section{1}

\section{Matériaux de l'étude}

Les essais ont été menés sur quatre sols reconstitués, composés de sable et d'argile en différentes proportions, pour lesquels l'application des différents critères d'effondrement, rapportés par Lutenegger et al. (1988), montrent que ceux-ci sont affaissables.

Le sable utilisé est un sable de dune lavé, ses caractéristiques sont résumées comme suit:

- granulométrie comprise entre $0,8 \mathrm{~mm}$ et $2,0 \mathrm{~mm}$, dont $1,5 \%$ en poids des particules est inférieur à $0,08 \mathrm{~mm}$; - coefficient d'uniformité 2,3;

- coefficient de courbure 0,91.

L'argile utilisée a les caractéristiques suivantes: - limite de liquidité $68 \%$;

- limite de plasticité 36\%:

- densité spécifique 2,7;

- pourcentage des particules inférieures à 2 microns : $76,4 \%$.

Les sols reconstitués ont les courbes granulométriques représentées sur la figure 1 et les caractéristiques géotechniques sont données dans le tableau I.

\section{2}

\section{Appareillage pour le compactage du matériau}

Le principe consiste à reconstituer un sol, dans le moule de l'cedomètre (Fig. 2), ayant une certaine teneur en eau et une certaine densité sèche. Pour cela, le matériau est compacté en deux couches au moyen d'un appareillage conçu au laboratoire. Cet appareillage, représenté sur la figure 3 , est composé d'un disque, ayant un diamètre légèrement inférieur à celui de l'anneau, fixé à une tige de guidage, et d'un mouton en forme de disque. Le mouton de masse $136 \mathrm{~g}$ coulissant le long de la tige tombe d'une hauteur de $15 \mathrm{~cm}$ et vient percuter le disque en compactant le matériau dans l'anneau de l'œdomètre.

TABLEAU I Caractéristiques physiques des sols utilisés dans cette étude.

\begin{tabular}{c|c|c|c|c|c|c|c|c} 
Sol & $\begin{array}{c}\text { Optimum } \\
\text { Proctor } \\
\text { W }_{\text {\%or }}\end{array}$ & $\begin{array}{c}\text { Densité } \\
\text { séche } \\
\text { max. }\end{array}$ & $\begin{array}{c}\text { Limite de } \\
\text { liquidité } \\
\mathrm{W}_{\mathrm{L}} \%\end{array}$ & $\begin{array}{c}\text { Limite de } \\
\text { plasticité } \\
\mathrm{W}_{\mathrm{p}} \%\end{array}$ & $\begin{array}{c}\text { Indice dé } \\
\text { plasticité } \\
\text { IP }\end{array}$ & $\begin{array}{c}\text { \% des } \\
\text { grains } \\
<2 \mu \mathrm{m}\end{array}$ & $\begin{array}{c}\text { Activité } \\
\text { (Ac) }\end{array}$ & $\begin{array}{c}\text { Densité } \\
\text { des } \\
\text { grains }\end{array}$ \\
\hline A & 7,5 & 2,01 & 18,5 & 13,5 & 5 & 6,2 & 0,8 & 2,7 \\
\hline B & 8,3 & 1,90 & 10 & - & - & 2,9 & - & 2,7 \\
\hline C & 10 & 1,80 & 8 & - & - & 1,2 & - & 2,7 \\
\hline D & 8 & 1,96 & 16 & 12,5 & 3,5 & 5,3 & 0,7 & 2,7 \\
\hline
\end{tabular}




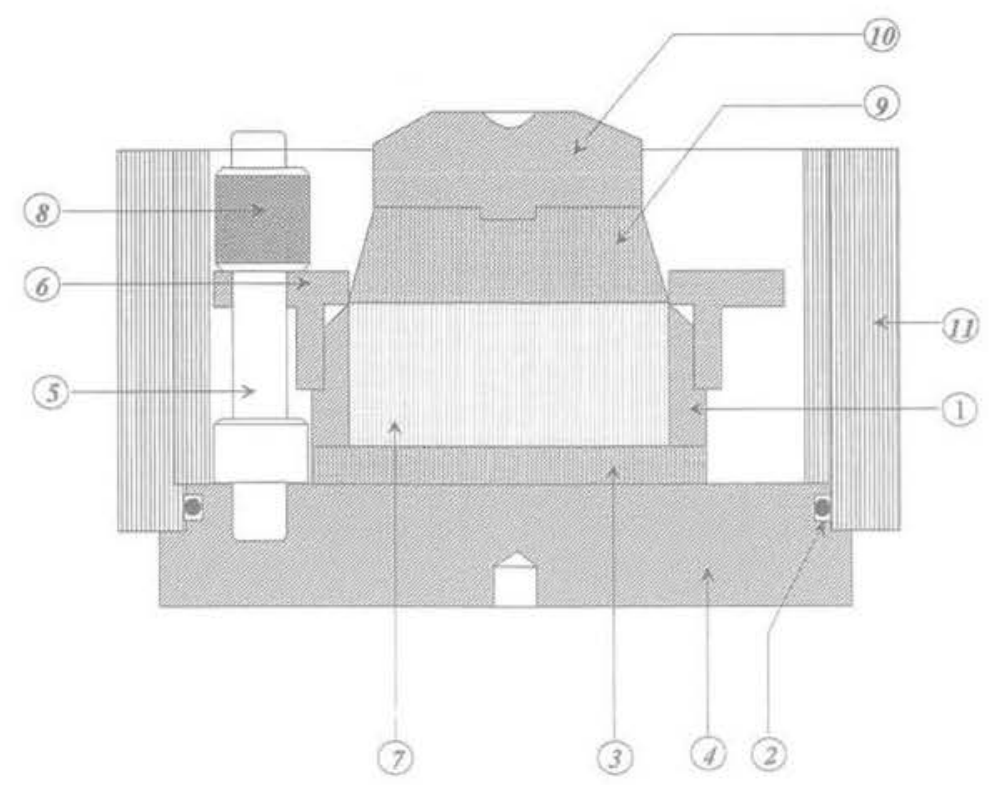

FG.2 Moule de l'œedomètre

1) Anneau rigide $(\Phi$ int $=50,47 \mathrm{~mm}$ ).

2) Joint étanche.

3) Disque poreux inférieur.

4) Bas du moule reposant sur le

bâti de l'œedomètre.

5) Plots (3) pour fixation.

6) Couvercle.

7) Échantillon.

8) Écrou de serrage.

9) Disque poreux supérieur.

10) Piston de chargement.

11) Paroi transparente.

Cellule of Oedometer.

1) Rigid ring.

2) O ring.

3) Bottom porous disc.

4) Cellule base

5) Fixation screws.

6) Lid.

7) Sample

8) Tiding nut.

9) Top porous disc

10) Loading piston

11) Transparent wall.

AG. 3 Dame de compactage.

repère

masse coulissante

tige de guidage

disque rigide

Hammer of compaction.

indication point

moving dead weight

guiding rod

rigid disc

\section{Fabrication de l'éprouvette et exécution de l'essai}

Les éprouvettes sont réalisées à partir d'un mélange de matériau de base (sable et argile) bien homogénéisé. Après homogénéisation, on amène le matériau à la teneur en eau voulue par ajout de la quantité d'eau distillée nécessaire.

Après préparation d'une gâchée bien homogénéisée, celle-ci est versée dans la trousse cylindrique de l'œdomètre puis compactée avec une certaine énergie

de compactage (nombre de coups de mouton/couche) au moyen du dispositif décrit ci-dessus. L'éprouvette est ensuite arasée au niveau du bord supérieur de l'anneau, à l'aide d'une lame rigide, pour obtenir une surface plane.

Après la pesée de l'éprouvette, celle-ci est remise dans le moule de l'œdomètre sur lequel sera effectué l'essai de compressibilité décrit par Jennings et Knight (1975).

L'essai consiste à charger le sol progressivement, à partir d'une teneur en eau et d'un poids volumique initial, jusqu'à $0,2 \mathrm{MPa}$, contrainte à laquelle on procède à l'inondation de l'échantillon. La déformation axiale de 
l'échantillon (tassement) a été mesurée par un comparateur ayant une précision de l'ordre de 1 micron. Pendant l'inondation, le tassement de l'éprouvette a été relevé à différents intervalles de temps.

\section{Programme d'essais}

Les essais sont effectués sur les sols à différentes teneurs en eau et différentes énergies de compactage. Les paramètres retenus sont:

- teneur en eau: $2 \%, 4 \%$ et $6 \%$;

- degrés de compactage: 10,20 et 30 coups/couche $(0,004 \mathrm{~kJ} ; 0,008 \mathrm{~kJ}$ et $0,012 \mathrm{~kJ}$ respectivement $)$.

\section{3}

\section{Résultats et discussion}

\section{1}

\section{Mise en évidence de l'effondrement des sols}

Après la préparation des sols reconstitués et la détermination de leurs propriétés physiques et mécaniques présentées dans le paragraphe précédent, on a effectué une série d'essais pour vérifier si ces sols sont vraiment affaissables et possèdent les propriétés connues des sols affaissables naturels.

Les résultats de ces essais pour le sol (A) sont montrés dans les figures 4 et 5 . Le potentiel d'affaissement CP a été calculé par la relation de Knight (1963):

$$
\mathrm{CP}=\frac{\Delta \mathrm{e}_{\mathrm{c}}}{1+\mathrm{e}_{\mathrm{o}}} \cdot 100 \%
$$

où :

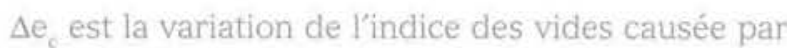
l'inondation et,

$\mathrm{e}_{0}$ est l'indice des vides initial.

(D'après la classification de Jennings et Knight (1975), l'affaissement ne se produit pas pour CP < 1\%.)

Une analyse de ces graphes, nous permet de déduire que:

- généralement, les sols partiellement saturés qui ont un poids volumique $\sec \left(\gamma_{\mathrm{d} 0}\right)$, inférieur à $15,5 \mathrm{kN} / \mathrm{m}^{3}$ sont susceptibles d'effondrement;

- pour une teneur en eau initiale donnée, le potentiel d'affaissement décroît quand on augmente l'énergie de compactage. Cette décroissance de CP est d'autant plus marquée que la teneur en eau augmente;

- les sols étudiés donnent des potentiels d'affaissement CP élevés pour des teneurs en eau initiales faibles (notamment quand ces sols, sont compactés à une teneur en eau très inférieure à l'Optimum Proctor); - pour une énergie de compactage donnée, l'affaissement diminue, quand la teneur en eau augmente.

Ceci est vrai aussi pour les sols (B), (C) et (D). Ces résultats rejoignent ceux de Barden et al. (1969) et Lawton (1989). On peut conclure que les sols qu'on a préparés artificiellement possèdent un comportement analogue à ceux rencontrés dans la nature, donc convenables pour le programme d'essais établi pour cette étude.

\section{2}

\section{Influence du coefficient d'uniformité sur l'amplitude de l'affaissement}

Pour élucider l'influence directe de la granulométrie sur l'amplitude de l'affaissement, on a représenté sur des graphes (Fig. 6 à 8), la variation du potentiel d'affaissement CP en fonction du coefficient d'uniformité U déduit à partir des courbes granulométriques de chaque sol utilisé dans les essais. En analysant les graphes $\mathrm{CP}=\mathrm{f}(\mathrm{U})$ et en se basant sur la classification de Jennings et Knight (1975) nous avons pu établir les valeurs du coefficient d'uniformité au-dessous desquelles il n'y a pas d'affaissement. Ces limites ont été regroupées au tableau II, en tenant compte des teneurs en eau initiales $(2 \%, 4 \%$ et $6 \%)$ ainsi que des niveaux d'énergie de compactage (10, 20 et 30 coups par couche).

TABLEAUII Valeurs du coefficient d'uniformité audessous desquelles il n'y a pas risque d'affaissement.

\begin{tabular}{c|c|c|c}
$\begin{array}{c}\text { Energie de compactage } \\
\text { nombre de coups/couche) }\end{array}$ & \multicolumn{3}{|c}{ Coefficient d'uniformité } \\
\hline 10 coups & 4 & 6 & 12 \\
\hline 20 coups & 5 & 12 & 30 \\
\hline 30 coups & 6 & 35 & 40 \\
\hline Teneur en eau initiale & $2 \%$ & $4 \%$ & $6 \%$ \\
\hline
\end{tabular}

En exploitant ces résultats et en considérant que les sols en question ont la possibilité de s'effondrer quand ils sont dans un état lâche ou peu compact avec un faible degré de saturation, on a conclu:

- si $U \geq 12 \quad$ : l'affaissement se manifeste;

- si $\mathrm{U} \leq 4 \quad$ : le risque d'affaissement est écarté; - et si $4<\mathrm{U}<12$ : domaine de transition où l'affaissement peut se produire.

Toutefois, on note que la limite supérieure du domaine de transition $(\mathrm{U}=12)$, doit être augmentée à 30 dans le cas des sols compacts, ayant une teneur en eau initiale $w_{0}$ voisine de l'Optimum Proctor normal dans le versant sec.

Pour valider ce résultat, nous avons recensé un certain nombre de sols affaissables connus, de différentes régions du monde et leurs potentiels d'affaissement (tests cedométriques). Il s'avère que la plupart de ces sols ont un coefficient d'uniformité $(U=d 60 / d 10)$ compris entre 10 et 30 , et sont caractérisés par un état lâche (porosité élevée) (tableau III).

Dans les travaux de génie civil, ce critère est facilement applicable et avantageux pour identifier les sols affaissables, puisque, d'une part il se base sur un seul paramètre qui est le coefficient d'uniformité déduit de la courbe granulométrique et, d'autre part, n'exige pas des échantillons non remaniés. En revanche, les autres méthodes prédictives qu'elles soient empiriques ou expérimentales utilisent plusieurs paramètres du sol et nécessitent souvent de grandes précautions, lors des prélèvements d'échantillons sur place, ainsi que leur manutention et leur conservation.

A noter que la plupart des sols affaissables essayés par nous ou mentionnés dans la littérature ont été 


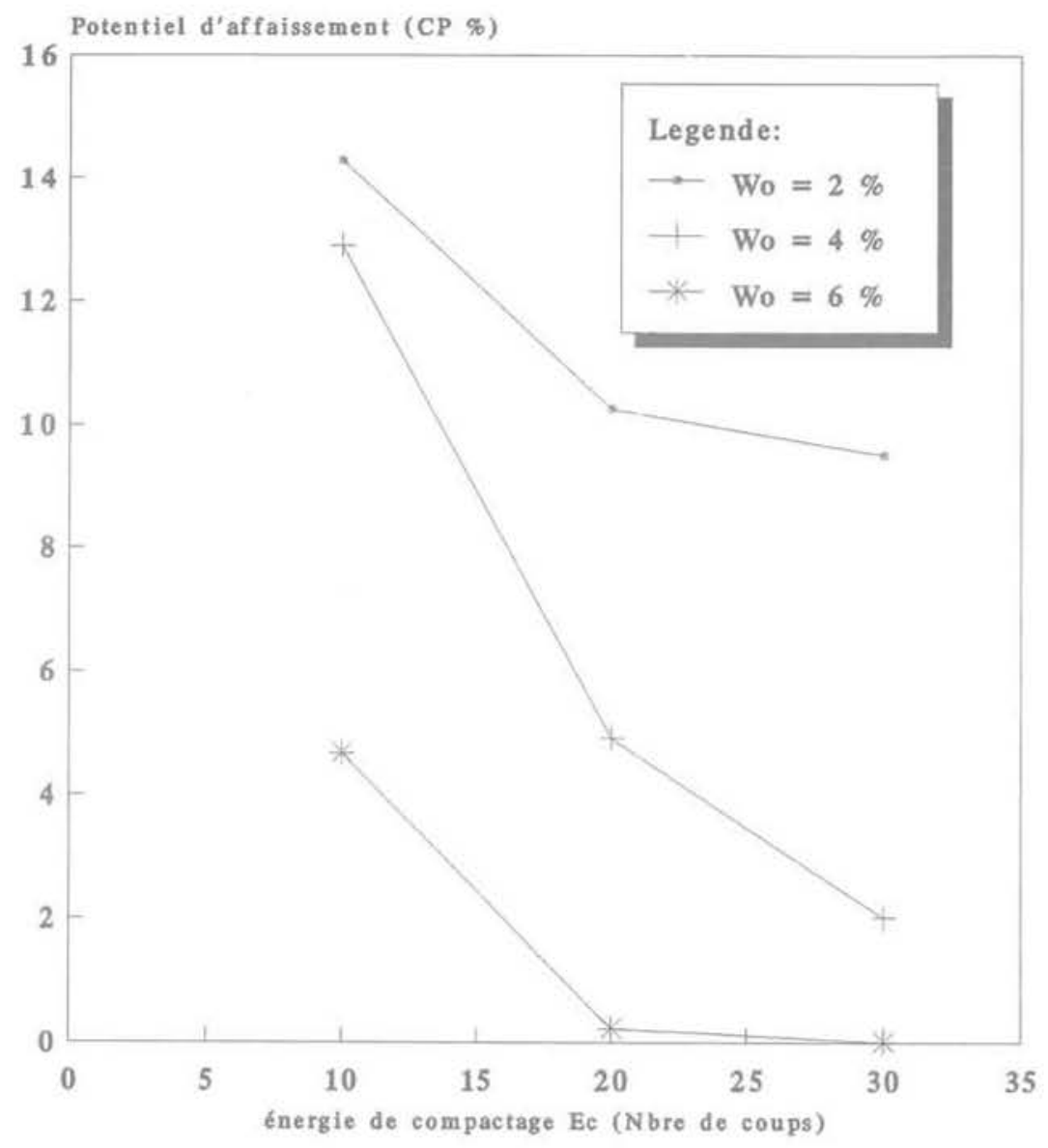

F16.4 Variation de CP en fonction de l'énergie de compactage pour le sol (A).

Collapse potentiel versus compacting energy for soil (A).

TABLEAUIII Exemples de sols affaissables avec leurs caractéristiques.

\begin{tabular}{|c|c|c|c|c|c|c|c|c|c|c|c|c|c|c|}
\hline Réference & Type de sol & $\begin{array}{l}\gamma_{j \mathrm{j}} \\
\mathrm{kN} / \mathrm{m}\end{array}$ & $e_{o}$ & n & $\begin{array}{l}\text { Wo } \\
\%\end{array}$ & $\begin{array}{l}\text { S, } \\
\text { of }\end{array}$ & $\mathrm{kN}_{\mathrm{N}}^{\gamma_{\mathrm{m}}}$ & $\begin{array}{l}W_{L} \\
\%\end{array}$ & $\begin{array}{l}\text { wp } \\
\%\end{array}$ & $\begin{array}{l}\text { IP } \\
\%\end{array}$ & $\begin{array}{l}\text { Ic } \\
\%\end{array}$ & Ac: & U & $\begin{array}{l}\text { CP } \\
\%\end{array}$ \\
\hline Ayadat (1995) & Sable argileux silteux & 15,4 & 72,1 & 41,9 & 4 & 14,5 & 2,65 & 20 & 13,5 & 6,5 & 2,46 & 0,72 & 106 & 13 \\
\hline $\begin{array}{l}\text { Lutenegger et al. } \\
\qquad \text { (1988) }\end{array}$ & Loess (Loveland) & 13,6 & 101,1 & 50,3 & 2,5 & 6,8 & 2.73 & 33 & - & - & - & - & 17 & 4.6 \\
\hline $\begin{array}{c}\text { Ferreira et al. } \\
\quad(1987)\end{array}$ & Sable argileux (Sâo-Polo) & 15,4 & 75,5 & 43 & 10 & 35,7 & 2,7 & 21 & 13 & 8 & 1,38 & 0,36 & 10 & 5,6 \\
\hline Yudhbir (1982) & Sol résiduel (Thaïlande) & 15,5 & 79,4 & 44,3 & 16 & 56 & 2,78 & 54 & 26 & 28 & 1,38 & - & - & 4 \\
\hline Yudhbir (1982) & Sol résiduel (Thaïlande) & 15,6 & 79 & 44,1 & 17 & 60 & 2,79 & 47 & 24 & 23 & 1,30 & - & - & 6.6 \\
\hline Zur et al. (1973) & Loess (Neguev) & 15,4 & 76,8 & 43,4 & 15 & 53,5 & 2,74 & 31 & 16 & 15 & 1,07 & - & - & 2 \\
\hline Dudley (1970) & $\begin{array}{l}\text { Sable argileux } \\
\text { (San Joaquin) }\end{array}$ & 15,1 & 79 & 44.1 & 6 & 20,5 & 2,7 & - & - & - & - & - & 28 & 5 \\
\hline
\end{tabular}




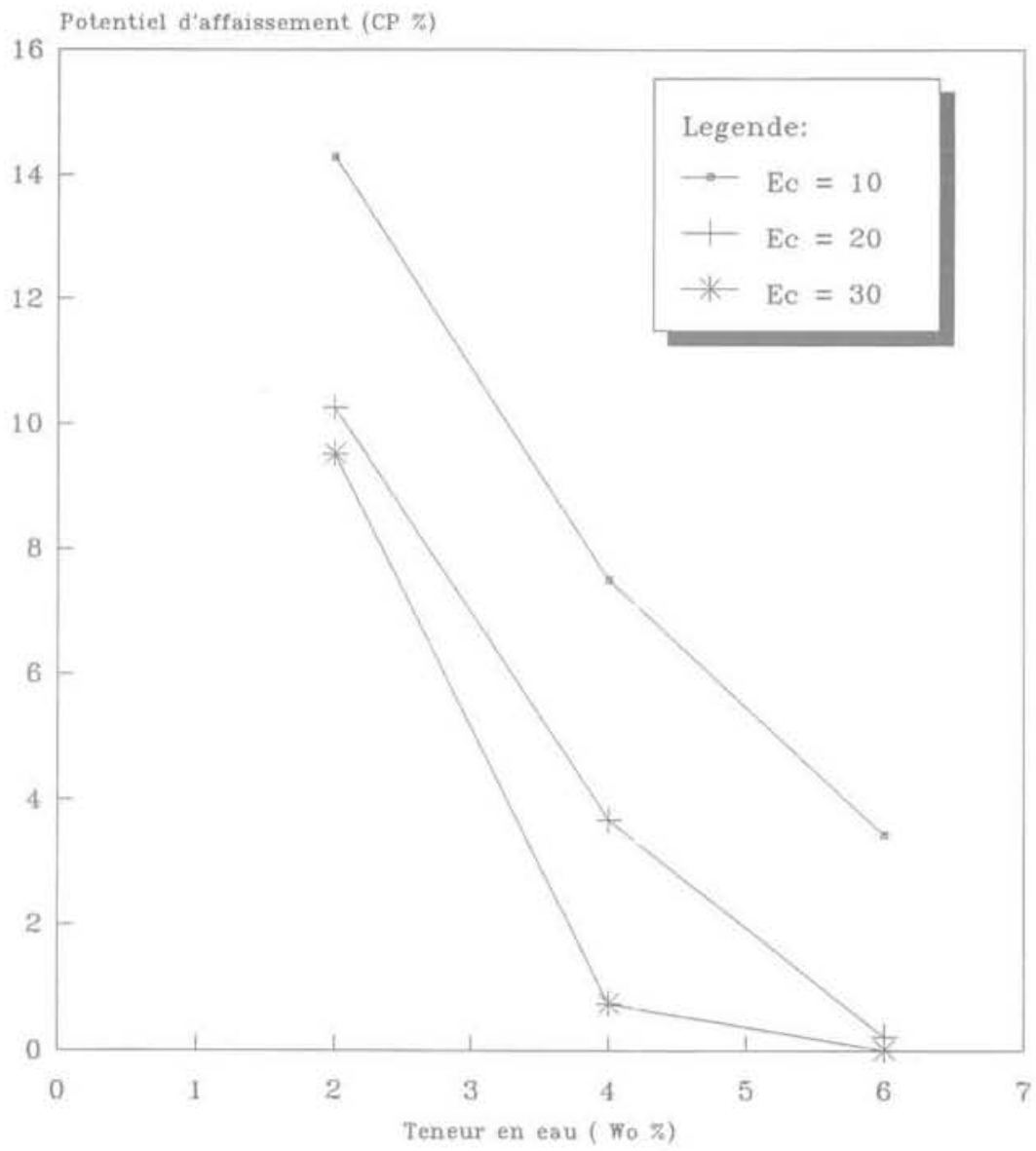

FIG.5 Variation de CP en fonction de la teneur en eau pour le sol (A).

Collapse potentiel versus compacting moisture content for soil (A).

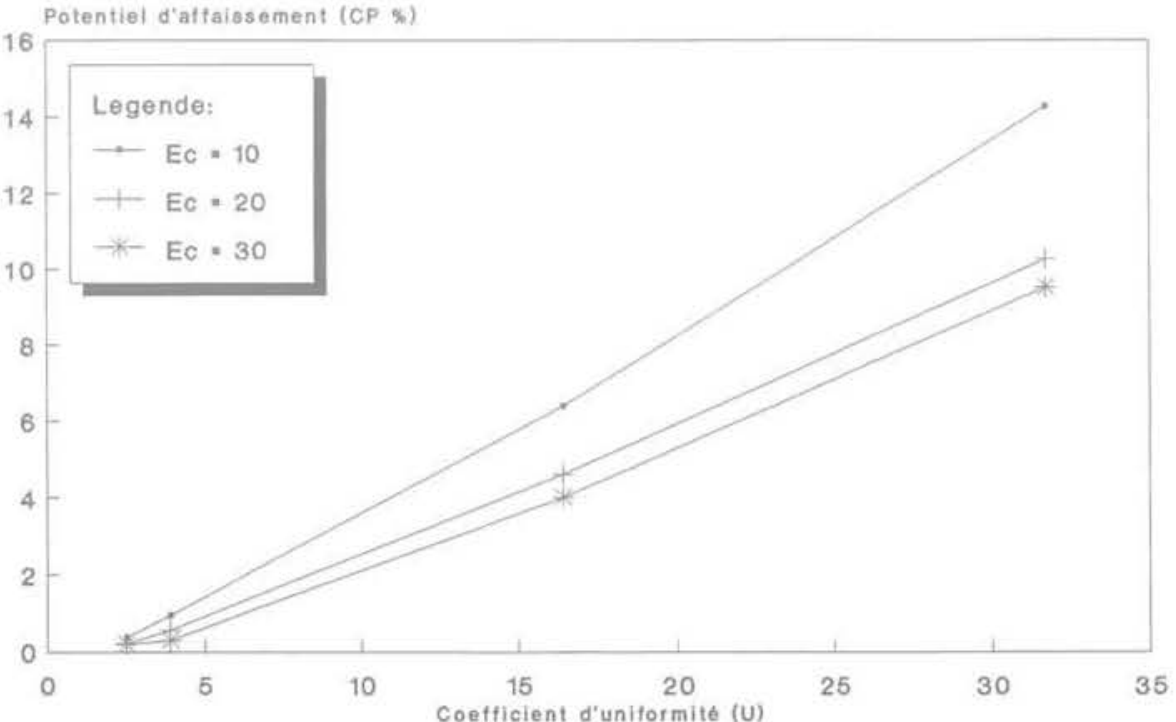

F10.6 Variation de CP en fonction du coefficient d'uniformité (pour $\mathrm{W}_{0}=2 \%$ )

Collapse potentiel versus uniformity coefficient (for $\mathrm{W}_{0}=2 \%$ ). 


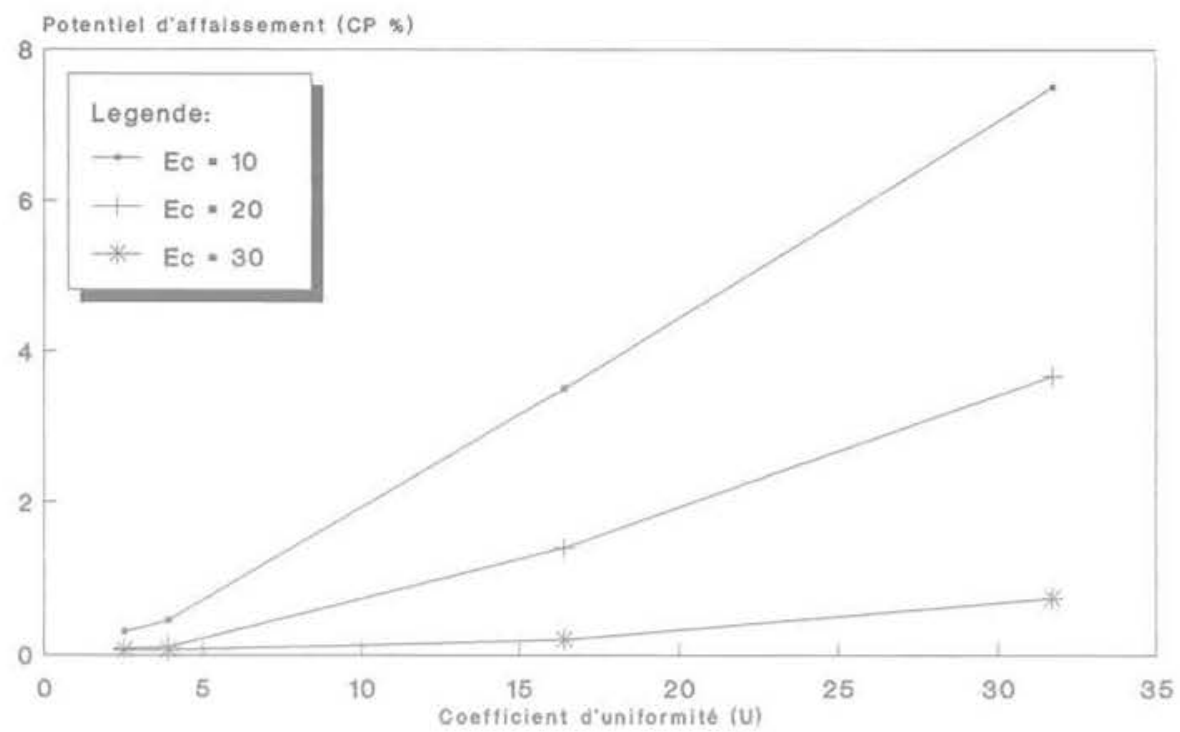

FG.7 Variation de CP en fonction du coefficient d'uniformité (pour $\mathrm{W}_{0}=$ $4 \%)$.

Collapse potentiel versus uniformity coefficient (for $\mathrm{W}_{0}=4 \%$ ).

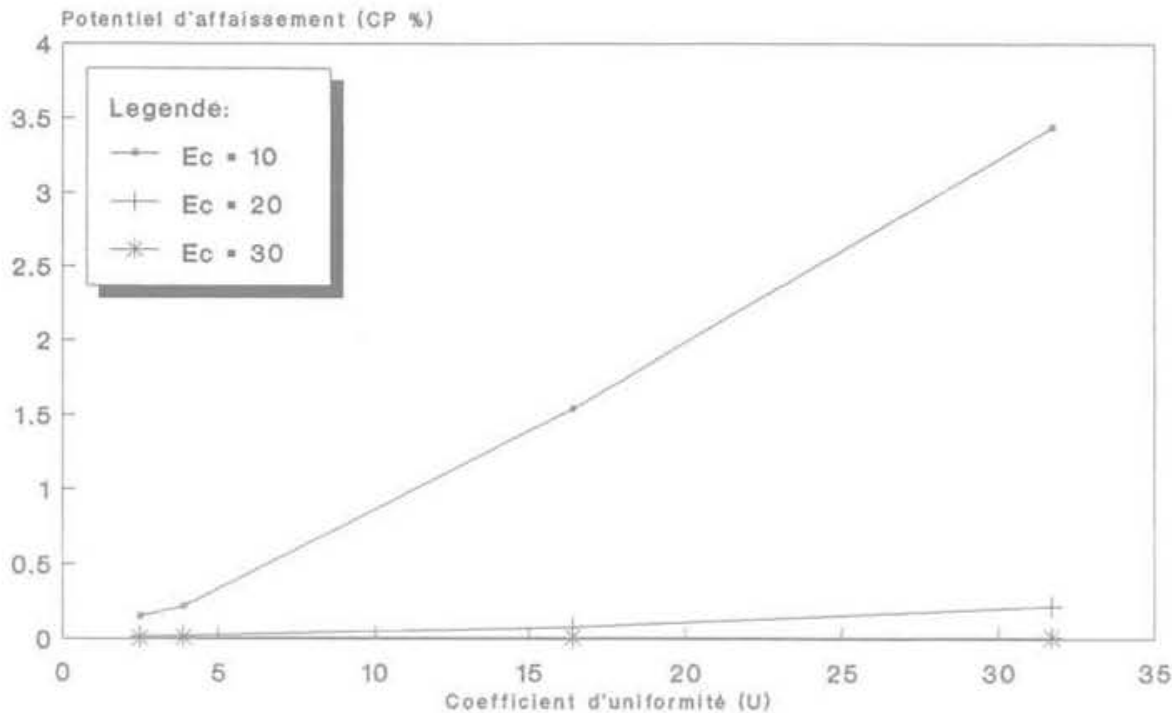

FG. 8 Variation de CP en fonction du coefficient d'uniformité (pour W0 = $6 \%$ ).

Collapse potentiel versus uniformity coefficient (for $W 0=6 \%$ ),

constatés inactifs. Nous croyons que c'est une condition nécessaire qui reste à vérifier.

\section{3}

\section{Influence du coefficient d'uniformité $U$ sur le degré d'affaissement}

On définit le degré d'affaissement DC, comme le rapport entre le potentiel d'affaissement à l'instant ( $t$ ) et le potentiel d'affaissement final; en d'autres termes c'est aussi le rapport entre le tassement à l'instant (t) et le tassement final. Il est exprimé par la relation suivante:

$$
\mathrm{DC}=\frac{\mathrm{Cp}(\mathrm{t})}{\mathrm{CP}} \cdot 100 \%
$$

$C P(t)$ : est le potentiel d'affaissement à l'instant $(t)$. $\mathrm{CP}$ : est le potentiel d'affaissement final.

Pour montrer l'influence de la granulométrie des sols sur le degré d'affaissement, on a représenté sur des graphes la variation de DC en fonction de U aux instants $\mathrm{t}_{1}=2 \mathrm{mn}$ et $\mathrm{t}_{2}=10 \mathrm{mn}$ (Fig. 9 et 10 ). On justifie le choix de ces deux valeurs du temps par le fait que l'essentiel du tassement s'est produit dans les dix premières minutes, pour les sols en question. D'après ces figures on peut faire les constatations suivantes:

a) à l'instant (t) donné, le degré d'affaissement DC augmente, quand le coefficient d'uniformité croît :

b) le degré d'affaissement DC décroît quand on augmente l'énergie de compactage, cette influence du compactage à faible teneur en eau initiale est très marquée pour les sols ayant une très faible teneur en argile (sols B et C) et insignifiante pour les sols possédant une plus grande teneur en argile (sols A et D). 


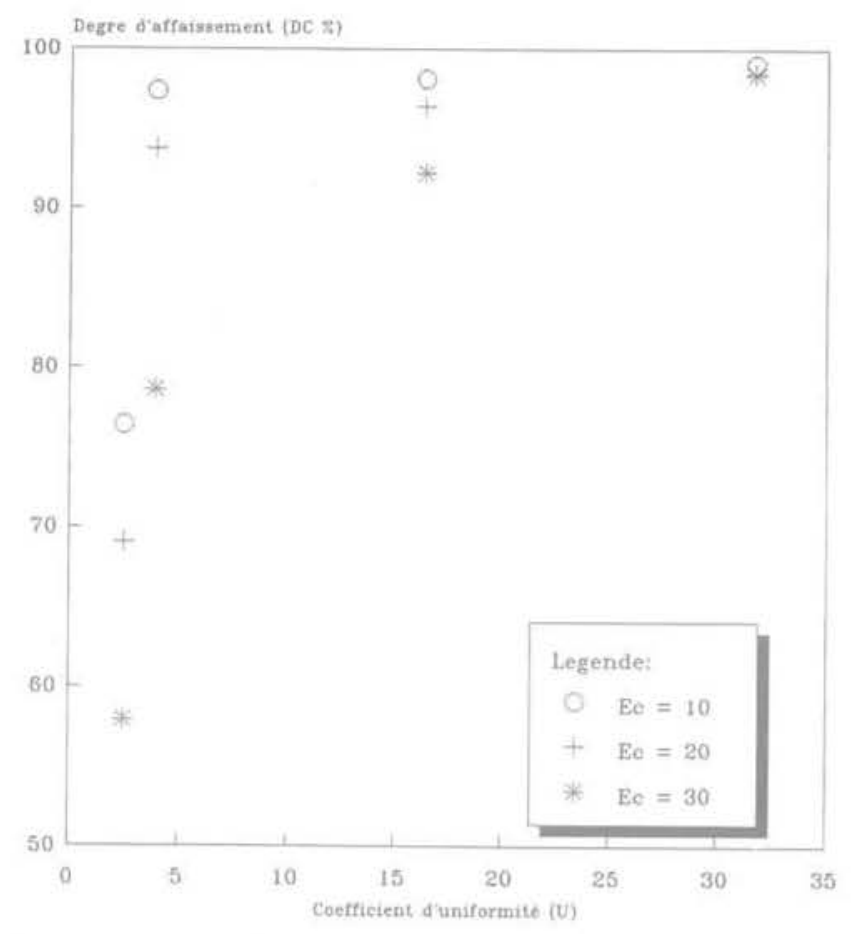

คG.9 Variation de DC en fonction de U (pour $\mathrm{W} 0=2 \%$ et $\mathrm{t}=2$ minutes). Degree of collapse versus uniformity coefficient for $\mathrm{WO}=2 \%$ and $\mathrm{t}=2 \mathrm{~min}$.

\section{Contribution à l'explication du phénomène d'affaissement}

\section{1}

\section{Quelques mécanismes proposés pour l'affaissement}

Un grand nombre de mécanismes d'affaissement ont été imaginés, d'après les résultats obtenus sur une variété de sols.

Knight (1961) a supposé que la résistance des liaisons argileuses entre les gros grains se réduisait par suite de l'addition d'eau à l'échantillon sous contrainte. Dès que cette résistance devient inférieure aux contraintes de cisaillement, il y a rupture des liaisons argileuses et I'affaissement ou l'effondrement se produit.

Barden et al. (1969) et Prusza, Choudry (1979) ont suggéré que l'affaissement est dû essentiellement à l'élimination de la succion capillaire présente dans ces sols qui sont le plus souvent partiellement saturés.

Clemence (1985), a indiqué que l'affaissement est très immédiat lorsque les particules sont liées par succion capillaire, lent dans le cas de la cimentation chimique et beaucoup plus lent dans le cas des liaisons argileuses entre les gros grains.

\section{4 .2}

\section{La migration des particules fines, comme approche d'explication du mécanisme de l'affaissement des sols}

Les résultats des essais (\$3.2 et 3.3) nous ont montré qu'il y a une dépendance entre granulométrie et affais-

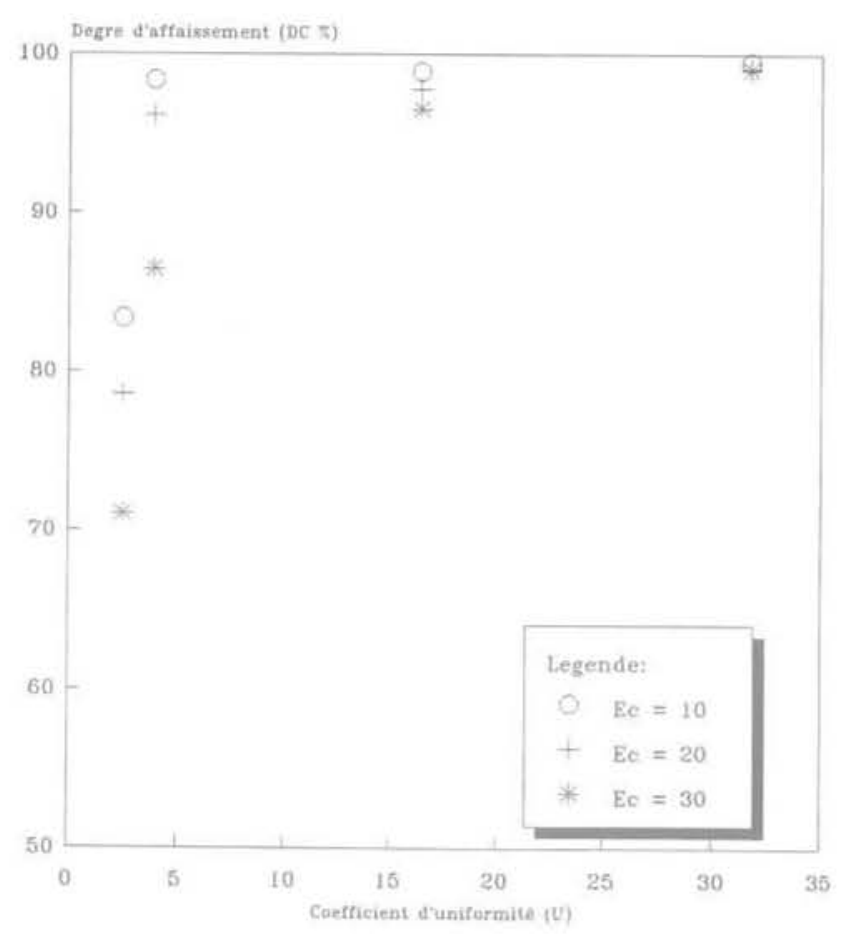

FIG, 10 Variation de DC en fonction de U (pour $\mathrm{WO}=2 \%$ et $\mathrm{t}=10$ minutes). Degree of collapse versus uniformity coefficient for $W O=2 \%$ and $t=10 \mathrm{~min}$.

sement. Cette sujection peut être envisagée pour contribuer à l'explication du mécanisme d'effondrement des sols affaissables en utilisant la notion de suffusion ou migration des particules fines dans la structure de ces sols, lorsque ces derniers sont inondés à l'eau.

Hanna (1990), en exposant les problèmes causés par l'inondation des sols de fondation, a seulement avancé l'idée que le phénomène d'affaissement est dû à la migration des particules fines à travers le sol, d'un horizon ou d'un niveau à un autre; ce mécanisme est connu sous le norn de " suffusion ».

Istomina (1957) a donné la condition géométrique de la suffusion en termes de coefficient d'uniformité: $-\mathrm{U} \leq 10 \quad$ (pas de suffusion);

$-10<\mathrm{U}<20$ (zone de transition)

$-\mathrm{U} \geq 20$ (le sol est susceptible à la suffusion).

Cependant, dans la zone de transition, des gradients hydrauliques très élevés produisent la suffusion et lorsque $U>25$, les particules fines migrent aisément sous un faible gradient.

Les diverses observations précédentes montrent que l'idée avancée par Hanna, que l'affaissement des sols effondrables est lié à la migration des particules fines à travers la structure, se trouve ainsi confortée et serait en liaison avec le phénomène de suffusion.

\section{Conclusion}

Les conclusions principales qu'on peut tirer de cette étude se résument comme suit:

1) la formation artíficielle d'un sol affaissable, au laboratoire, s'obtient facilement, par le mélange de 
matériaux granulaires de différents calibres (gros grains, moyens et très fins), compactés avec une énergie modérée, à une teneur en eau inférieure à l'Optimum Proctor;

2) il y a une influence marquée de la densité sèche et de la teneur en eau, initiales, sur le comportement des sols affaissables. Il a été montré que, généralement, il y a des grands tassements, quand ces deux paramètres sont faibles:

3) il y a une influence de la granulométrie sur l'affaissement des sols qui sont dans un état lâche ou peu compact avec un faible degré de saturation. Si le coefficient d'uniformité $U \geq 12$, l'affaissement est possible et, si $U \leq 4$, il n'y a pas d'affaissement. Entre ces deux limites, l'affaissement est seulement probable (zone de transition)

4) le potentiel d'affaissement CP et le taux d'affaissement DC augmentent, quand le coefficient d'uniformité croît;

5) le phénomène de suffusion ne doit pas être écarté quand on recherche les mécanismes d'affaissement. On peut penser que l'effondrement de ces sols sous mouillage est causé par la migration des particules fines, après élimination de la cohésion apparente.

\section{$\overline{\text { Bibliographie }}$}

Anderson P.J. - «Determination of collapse potential of solls x (reporté par Lutenegger A.J. et Saber R.T.J, Geot. Testing Jnl, GTJODJ, vol, 11, $n^{\circ} 3,1988$, p. $173-178$

Ayadat T. - « Collapse of stone column foundation due to inundation $x$, a paraitre dans la revue Journal of Technology (Algérie), 1995.

Barden L. Madedar A.O., Sides G.R. «Volume change characteristics of unsaturated clayn, Journal of SMFD, ASCE, vol. 95, SM1, 1969, p. 33-49.

Booth A.R. - "Collapse settlement in compacted soils \$, CSIR Research Report 324. NITRR bulletin 13, Pretoria, South Africa, 1977

Bull W.B. - "Alluvial fans and near-surface subsidence in Western Fresno county California ». Professional Paper, 4-37-A United States Geological survey, Washington D.C., 1964, p. 72

Clemence S.P. - «Collapsible soils : identification, treatment and design considerations $n$. Current Practices in Geotechnical Engineering, vol.1, Geo-Environ Academia, 1985

Dudley J.H. - «Review of collapsing soils $w_{\text {, }}$ Journal of Soil mechanics and Founda- tions Division, ASCE, vol. 96, n* SM3 Proc. Paper 7278, May 1970, p. 925-947.

Ferreira R.C. Peres J.E., Monteiro L.B. "Some characteristics and properties of collapsible brazilian soils x, Proceeding of the int. Symposium on Geotechnical Eng. of Soft Solls, Mexico, 1987, p. 39-44.

Handy R.L. - «Collapsible loess in Lowa ». Soil Science Society of America Proceeding, vol. 37, 1973, p. 281-284.

Hanna T.H. - «Foundation difficulties - an overviewn, 4th Indonesian Conference for Geotechnical Engineering, Bandung, February 26-27, 1990

Istomina V.S. - "Filtration stability of soils n (in Russian), Gostroizdat, Moscow, Leningrad, 1957

Jennings J.E.. Knight K. - "A quide to construction on or with materials exhibiting additional settlement due to collapse of grain-structure 7 , Proc. 6th Regional Conf. For Africa on SMFE, Durban. South Africa, 1975.

Knight K. - a Collapsing behaviour of residual solls n (reporté par Yudhbir), Proceeding of the 7 th Southeast Asian Geotechnical Conference, Hong Kong, November 1982, p. 915-930

Knight K. - " The origin of occurence of collapsing soils 10 , Proc 3rd Regional Conf. for Africa on SFME, vol. 1, 1963 p. $79-94$.

Lawton E.G., Fragaszy R.J., James H.H. "Collapse of compacted clayey sand $n, J$. of Geol. Engng Div., ASCE, vol. 115, n 9 , 1989, p. $1252-1267$

Lutenegger A.J., Saber R.T. - «Determination of collapse potential of soils m), Geot. Testing Jnl, GTJODJ, vol. 11, n³, 1988, p. 173-178.

Markin B.P. - Díscussion on \& Standand criteria of sag in loess soilsm, ASCE, Soil mech. and found. Engng, n² 2. 1969, p. 137.

Prusza A. Choudry T. $-\alpha$ Collapsibility of

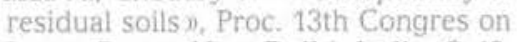
Large Dams, New Delhi, India, Q.49, R.9, 1979, p. $117-130$.

Yudhbir - "Collapsing behaviour of residual soils $n$. Proceeding of the 7 th Southeast Asian Geotechnical Conference, Hong Kong, November, p. 915-930,

Zur A., Wiseman C. - " Study of collapse phenomena of an undisturbed loess p. Proceeding 8th Int. Conf. of soil mech. and Found. Eng. Moscow, USSR, vol. 2, part 2, 1973, p. 265-269. 